\title{
LA UTILIZACION DE LOS ESPACIOS VERDES EN LA CIUDAD DE LOGROÑO ${ }^{1}$
}

\author{
Montserrat Muga Fernández
}

El tipo de crecimiento experimentado en el último siglo por las ciudades y la mayor disponibilidad de tiempo libre por parte de sus habitantes han hecho de los espacios verdes un tema de gran actualidad.

No podemos dar una definición clara y precisa de lo que se entiende por espacios verdes, ya que no es un concepto estático sino en constante evolución como la misma sociedad. Se podrían definir las zonas verdes como zonas públicas, libres de edificación, donde las personas mantienen unas relaciones sociales, pasean, descansan o disfrutan de su tiempo de ocio. En nuestro estudio sobre la ciudad de Logroño no entendemos por espacios verdes solamente aquellas zonas que tengan árboles o césped, pues también hemos incluido las áreas de esparcimiento que aunque no dispongan de un sólo árbol, por sus características permiten una utilización parecida a la de los espacios verdes propiamente dichos. Por esta razón asimilamos aquí espacios verdes a áreas de esparcimiento y a espacios libres. De hecho, si no hubiéramos partido de este principio hubiera sido prácticamente imposible llevar a cabo un estudio como éste en la ciudad de Logroño.

Nos interesa la utilización de los espacios verdes en función de su situación en el conjunto de la ciudad y en función asimismo de una serie de variables coyunturales que condicionan la participación de los ciudadanos en las áreas de esparcimiento. Sin embargo consi-

1. El presente trabajo es un resumen de la Tesis de Licenciatura de la autora que con el título de "Los espacios verdes de la ciudad de Logroño" fue leida en septiembre de 1980 en la Facultad de Filosofía y Letras de la Universidad de Zaragoza. La dirección corrió a cargo del Dr. D. José M.a García Ruiz y se realizó en el seno del Departamento de Geografía del Colegio Universitario de Logroño. 
MONSERRAT MUGA FERNANDEZ

deramos de interés realizar antes un breve esbozo de las características climáticas de Logroño, con varios objetivos:

1.- Conocer las dificultades de mantenimiento de los espacios ajardinados.

2.- Tener una idea básica acerca de la intensidad con que pueden utilizarse los espacios verdes a lo largo del año y a lo largo del día.

3.- Deducir algunas de las características de que debieran estar dotados los espacios verdes desde una óptica climática.

\section{A) El clima de Logroño como condicionante del uso de los espacios vercies.}

Logroño se localiza a caballo entre la Rioja Alta y la Rioja Baja, que aunque incluidas dentro de un mismo dominio climático, participan de algunas diferencias de matiz. Mientras que la Rioja Alta disfruta de temperaturas más suaves y lluvias más abundantes (en Haro $504 \mathrm{~mm}$ ), a medida que descendemos hacia el Este las temperaturas aumentan y las lluvias disminuyen (en Alfaro $374 \mathrm{~mm}$ ), pues la influencia mediterránea está más próxima. En Logroño influyen quizá más las características climáticas de la Rioja Alta, sobre todo por la sucesión de tipos de tiempo.

Los datos que hemos utilizado han sido tomados del Observatorio Metereológico del Aeródromo de Agoncillo situado a $2^{\circ} 19^{\prime}$ $40^{\prime \prime} \mathrm{W}$ de longitud y $42^{\circ} 27^{\prime} 00^{\prime \prime} \mathrm{N}$ de latitud; la altitud de la estación es de 353 metros y está localizada en las proximidades de la capital. Los datos se basan en series de treinta años que van desde 1948 a 1977, a partir de los cuales se han elaborado las medias (Sánchez-Gabriel y Fernández Giró, 1979).

La precipitación media anual en Logroño es de 402,0 litros $/ \mathrm{m}^{2}$; el mes de mayor pluviosidad es junio con $45,4 \mathrm{litros} / \mathrm{m}^{2}$, seguido de mayo con 43,8 litros $/ \mathrm{m}^{2}$ y septiembre con 41,0 litros $/ \mathrm{m}^{2}$. Como podemos ver las lluvias son equinocciales, siendo julio el mes de menores precipitaciones, con 20,8 litros $/ \mathrm{m}^{2}$, en tanto los meses de enero y febrero presentan un pequeño mínimo, no tan acusado como el estival. El régimen de precipitaciones responde aun recrudecimiento del paso de corrientes frontales procedentes del Atlántico tanto en primavera como en otoño; en esta última estación interviene también la arribada de vientos procedentes del Mediterráneo, ligados a 


\section{LOS ESPACIOS VERDES DE LOGROÑO}

a pequeñas pero intensas depresiones que se forman en el Mediterráneo occidental desde finales del verano. En invierno, el descenso de precipitaciones se debe a la implantación de un pequeño anticiclón sobre la Península, lo que dificulta la entrada de frentes; mientras en verano el anticiclón de las Azores domina extensamente en el suroeste de Europa y origina un período de sequía tanto más prolongado cuando más al Sur.

Las nevadas apenas tienen importancia y desde luego la nieve es muy poco persistente. Lo que si hay que reseñar es que la disposición del relieve favorece las inversiones térmicas, lo que produce aproximadamente unos cuarenta días al año de niebla, siendo enero el mes donde estos días son más numerosos, seguido de diciembre y noviembre. Estas inversiones térmicas que favorecen la formación de nieblas son muy frecuentes en todo el valle del Ebro.

La nubosidad media es de $5 / 8$, siendo los meses más nubosos diciembre con $6 / 8$ y enero; por el contrario el mes menos nuboso es julio con una media de $3 / 8$. En contrapartida el promedio anual de horas de sol es de 2.345 horas y 25 minutos; julio es el mes más soleado, con 290 horas y 47 minutos y el mes menos soleado es diciembre, con 99 horas y 44 minutos.

Los vientos predominantes son del W y NW. Durante el verano son frecuentes los vientos de componente E, procedentes del Mediterráneo: el Bochorno, viento muy cálido que da lugar a temperaturas elevadas y una sensación de agobio.

La temperatura media anual es de $13,3{ }^{\circ} \mathrm{C}$. Enero y febrero son los meses más fríos; por el contrario es julio el mes más caluroso con una temperatura media de $21,9{ }^{\circ} \mathrm{C}$ seguido de agosto con una media de $21,6^{\circ} \mathrm{C}$.

Una vez vistas las características medias del clima conviene presentar un rápido esquema de los tipos de tiempo que se suceden en las estaciones del año, con lo que tendremos una idea más aproximada de la utilización de los espacios verdes.

a) En invierno las temperaturas descienden frecuentemente por debajo de cero grados, si bien durante el día es difícil que persistan las heladas. Durante diciembre, enero y febrero se produce una sucesión de días brumosos, con nieblas muy poco agradables, y días nublados que descargan alguna precipitación no muy abundante. 
Por otra parte, es normal que algunos de los días de niebla matinal se conviertan luego en soleados y apacibles. Normalmente no son meses muy ventosos, por lo menos hasta mediados de febrero.

b) En primavera el incremento térmico es bien constatable. Desaparecen las nieblas y alternan días húmedos, con precipitaciones frecuentes y a veces intensas, incluso vientos violentos que pueden ser fríos, con días más calmados, situación anticiclónica, tiempo soleado y agradable. El tiempo cambia con mucha frecuencia lo que no deja de ser la característica más singular de la estación.

c) Desde mediados de Junio las precipitaciones se hacen cada vez más raras (quizá algún frente roza levemente por La Rioja en la primera quincena de julio). En agosto y septiembre no son raras las tormentas, que descargan con gran violencia en un corto espacio de tiempo. El predominio corresponde a días soleados, muy calurosos, con temperaturas agradables hasta las $10 \mathrm{u} 11$ horas de la mañana o después de las 9 de la noche; el resto del día soporta temperaturas superiores a los $33-35{ }^{\circ} \mathrm{C}$. a la sombra, sin que apenas corra la más mínima brisa de aire.

d) A partir de mediados de septiembre las temperaturas se suavizan. Son quizá los mejores meses del año, hasta mediados de noviembre. En octubre irrumpen de nuevo las precipitaciones aunque no de forma tan continuada ni intensa como en primavera.

Como resultado podemos clasificar a La Rioja -y dentro de ella a la ciudad de Logroño- con un clima mediterráneo-continentalizado matizado entre La Rioja Alta y La Rioja Baja. Aplicando el criterio de LANG, el clima de La Rioja se nos presenta como árido, y según el método de THORNTHWAITE, como un clima semiárido con vegetación esteparia, si bien se encierra próximo al límite correspondiente al clima sub-húmedo.

En general todas las zonas verdes de Logroño participan de las características climáticas de la ciudad. Solamente se da la excepción del Parque del Carmen donde subsisten especies que no llegarían a desarrollarse fuera del parque. Incluso en la Glorieta del Doctor Zubía, debido a que todos sus árboles tienen un gran porte, durante el verano desciende la temperatura $1^{\circ}$ e incluso $2^{\circ} \mathrm{C}$, con respecto a la existente en la ciudad.

Así pues, desde un punto de vista climático y en relación con las zonas verdes podemos extraer las siguientes conclusiones: 


\section{LOS ESPACIOS VERDES DE LOGROÑO}

a) Los espacios ajardinados encuentran dificultades de conservación desde mediados de junio hasta finales de septiembre e incluso más si el otoño comienza seco. Es una época en que las precipitaciones descienden a niveles muy bajos y las temperaturas aumentan la evapotranspiración. Sin la ayuda de riegos periódicos es imposible el mantenimiento de espacios ajardinados. Por otro lado esto hace imposible -o al menos muy difícil-que pueda pensarse en el establecimiento de grandes zonas acespedadas en el futuro. El resto del año, aunque las precipitaciones no son excesivamente abundantes, las temperaturas son más bajas y la vegetación no tiene tantas exigencias en humedad.

b) Dadas las características climáticas de Logroño puede afirmarse la posibilidad de una utilización relativamente intensa de sus espacios verdes. Como es lógico, el invierno es la peor estación, por la frecuencia de días fríos, húmedos y brumosos, pero aún así pueden aprovecharse una serie de días que, aunque fríos, son soleados y calmados. En primavera aumentan los días buenos y agradables, y mucho más aún en otoño, época en que no son muy frecuentes los días lluviosos. En cualquier caso, en ambas estaciones, si el día es agradable puede hacerse uso de los espacios verdes durante toda la jornada, desde las $10 \mathrm{u} 11$ horas de la mañana hasta las 7-8 de la tarde. En verano la situación es más compleja, pues a mediodía el calor es demasiado agobiante. Aun así, los pocos espacios verdes en Logroño son los únicos enclaves de la ciudad donde puede soportarse medianamente el rigor estival. La mayor duración de la luz solar amplía en esta época el aprovechamiento de los espacios libres, si bien cabe señalar que entre las 2 y las 6 de la tarde se encuentran muy poco concurridos por la elevación de las temperaturas y el descanso posterior al almuerzo de mediodía.

c) La planificación de los futuros espacios verdes debe tener en cuenta la necesidad de dejar algunos rincones sin arbolado, expuestos al Sur, Este y Oeste, con objeto de que sus usuarios -sobre todo los ancianos- puedan disponer de un sector soleado y más cálido en invierno. A la vez, en otros sectores debe reforzarse la plantación de arbolado frondoso (sauces y plátanos, algunas acacias a condición de que se pongan muy juntas), con algunas fuentes, en tramos más umbríos, donde el máximo calor estival pueda verse en parte superado. 


\section{MONSERRAT MUGA FERNANDEZ}

\section{B) E1 uso de los espacios verdes por la población de Logroño}

Para ver la utilización que de los espacios verdes existentes en la ciudad de Logroño hacen sus habitantes, empleamos la metodología de la encuesta consistente en acudir a estas zonas en diferentes horas, días y con distinta climatología.

Las horas en las que se ha acudido van desde las diez de la mañana hasta la diez de la noche y los días analizados incluyen todos los de la semana. La climatología incluye días de lluvia, días con abundante nubosidad y días completamente despejados, todos ellos con diferentes temperaturas. Las estaciones analizadas abarcan las cuatro en las que se divide el año.

En las encuestas se diferencian por sexos y se agrupan por intervalos de edad. Los grupos los hemos dividido del siguiente modo: menores de diez años, entre diez y quince, quince-veinte, veintetreinta, treinta-cuarenta, cuarenta-cincuenta, cincuenta-sesenta, sesenta-setenta y más de setenta.

Los fines que hemos pretendido con estas encuestas son:

a) Ver qué tipo de personas utilizan con más asiduidad las zonas verdes.

b) Qué horas y qué días son los más concurridos.

Todo este problema es muy importante, ya que en función de las personas que acuden, las zonas verdes revestirán unas características $u$ otras, aunque se tiende a que estas zonas acojan a todo tipo de personas en sus diferentes edades y actividades.

Los espacios verdes analizados son todos los existentes, aunque se han analizado de forma preferente dos: El Espolón y el parque de Gallarza debido a sus especiales características.

E1 Espolón ha sido seleccionado por su extensión y sus condiciones estéticas, pero sobre todo por su situación en el interior de la ciudad y su tradición como la mejor área de esparcimiento de Logroño. A él acuden numerosas personas por la centralidad de que disfruta.

Por su parte, el parque de Gallarza se ha seleccionado por encontrarse al Sur de la ciudad, en un sector que se ha extendido mucho en los últimos años y carece de otras zonas verdes, lo que hace que su utilización sea masiva. Por otra parte, al tratarse de un sector relativamente nuevo, es muy numerosa la población infantil. 


\section{LOS ESPACIOS VERDES DE LOGROÑO}

En páginas adjuntas y en los apéndices finales se incluyen una serie de cuadros-resumen seleccionados de entre todas las encuestas realizadas. En ellos pueden apreciarse la proporción por edades de los visitantes y las diferencias existentes entre días con situaciones climáticas distintas y entre los dos parques analizados.

Comenzando por los resúmenes totales podemos deducir ${ }^{2}$ :

1. ${ }^{\circ}$ Una mayor presencia en ambos parques de población muy joven o muy mayor, es decir, personas que disponen de mucho tiempo libre. La frecuencia entre los hombres va disminuyendo de los menores de 10 años hasta alcanzar su mínimo entre los de 40 a 50 años, para a partir de ahí recupearse rápidamente entre los 60 y 70 años, con una clara inflexión a partir de esa edad. Entre las mujeres, la curva de distribución es algo más irregular, con un engrosamiento entre los 20 y 30 años. La diferencia de curva entre los hombres y las mujeres responde al hecho de que la mayoría de los niños que acuden al parque van acompañados por sus madres, en edades no muy posteriores a los 25 años.

$2 .^{\circ}$ Un predominio de hombres sobre las mujeres en el conjunto de los asistentes y en la mayoría de las clases (excepto de 20 a 60 años porque es la edad de máxima participación en el trabajo entre los hombres, mientras que entre las mujeres esa participación es sensiblemente menor). Tal predominio de hombres se hace más patente en las edades superiores, donde paradójicamente la estructura demográfica de la ciudad presenta una situación inversa. Ello se explica, no obstante, por el hecho de que los jubilados ya no tienen ninguna ocupación y están libres para acudir a los parques, mientras que las mujeres mayores aún desempeñan trabajos domésticos.

3..$^{\circ}$ Entre el Espolón y el parque de Gallarza aparecen también interesantes diferencias. Para empezar, el número de personas que acuden al Espolón es notablemente superior, lo que se explica por la mayor extensión y mayor capacidad de atracción de la más tradicional área de esparcimiento de Logroño.

4. ${ }^{\circ}$ Pero sobre todo, el parque de Gallarza presenta una estructura demográfica entre sus asistentes más rejuvenecida que la del

2. Ciertamente en los resúmenes totales se han sumado encuestas pertenecientes a horas y condiciones climáticas, lo que resta solvencia a los resultados finales. Con todo, como esquema comparativo, a título de orientación, creemos que tiene interés el análisis de tales resultados. 


\section{MONSERRAT MUGA FERNANDEZ}

Espolón. No hay que olvidar aquí varios hechos:

a) El parque de Gallarza se encuentra en un sector urbano de expansión relativamente reciente, con localización mayoritaria de matrimonios jóvenes, lo que aumenta el número de niños.

b) El Paseo del Espolón con situación central y con influencia sobre casi toda la ciudad, se asienta en un sector ya tradicional de Logroño, junto al Casco Viejo, donde predomina la población ya mayor.

c) El Espolón resulta más tranquilo para los ancianos que el parque de Gallarza, frecuentemente invadido por niños por la proximidad de un colegio.

Si descendemos a un nivel más detallado de los datos, analizando la respuesta de la población ante situaciones climáticas concretas, los resultados anteriores se confirman, pudiendo llegarse a las siguientes conclusiones:

1) En todas las situaciones analizadas es superior el número de hombres que el de mujeres, si bien parece comprobarse que las diferencias entre unos y otros se atenúan en los días agradables y despejados. En los días fríos la participación de los primeros es netamente superior (por ejemplo, el día 3-1-1980 en el paseo del Espolón).

2) De igual forma predominan siempre las mujeres comprendidas entre los 20 y los 60 años, si bien su superioridad numérica es mucho menor en los mejores días, contra lo que pudiera esperarse. Parece como si la mujer de esa edad estuviera acostumbrada a salir con sus hijos con relativa independencia de la situación climática, mientras el hombre de esa edad sólo va al parque si el tiempo es relativamente bueno (por ejemplo, el día 15-6-1980 en el Paseo del Espolón). En días fríos, aunque el total de personas que acuden al parque disminuye considerablemente, las mujeres de 20 a 50 años presentan una participación muy aceptable.

3) La situación climática es determinante a la hora de analizar la afluencia de personas a los parques riojanos, conclusión que era perfectamente previsible a priori. De todos los tipos de tiempo el menos aceptable corresponde a las nieblas (como lo prueba el resultado del día 4-12-1979 en el Paseo del Espolón; donde se contabilizaron 43 personas en total, 25 hombres y 18 mujeres), seguido de los días nublados y fríos. Sin embargo la población acude a los parques con bastante intensidad en días de cielo despejado aunque fríos. 


\section{LOS ESPACIOS VERDES DE LOGROÑO}

4) La máxima afluencia coincide siempre entre los hombres de más de 60 años, aunque las condiciones climáticas sean desagradables. En esta última circunstancia se da el caso de que al disminuir el número de niños aumenta la participación de los ancianos; y así el día 4-12-1979, con abundante niebla, este grupo de personas representó el 60 por 100 del total. No sucede lo mismo entre las mujeres, que disminuyen notablemente en días desapacibles y fríos al no poder salir con los niños pequeños.

5) No hay una clara participación joven en los parques. El grupo comprendido entre los 15 y 20 años no aporta nunca cifras elevadas. Unicamente se aprecia un ligero incremento durante las vacaciones escolares, pero sin que pueda hablarse de aumentos muy significativos.

6) Igualmente existe una variación en cuanto a los días de la semana, siendo el sábado y sobre todo el domingo los días de mayor afluencia de personas, a la vez que es durante estos días cuando personas en edad de trabajar acuden a los parques en compañía de sus hijos e incluso se pueden ver a grupos de jóvenes.

Existen en la ciudad diferentes zonas que a pesar de no ser propiamente zonas verdes atraen a un número relativamente importante de personas. Nos referimos a la Plaza del Mercado y a la plaza de San Bartolomé. La Plaza del Mercado, ocupada en gran parte por un aparcamiento para coches, a pesar de sus reducidas dimensiones posee unos cuantos bancos que frecuentemente están ocupados por personas mayores. Lo mismo ocurre en la Plaza de San Bartolomé. Ambas están enclavadas dentro del Casco Antiguo, sector con un alto índice de envejecimiento, por lo que las personas mayores encuentran dificultades a la hora de desplazarse; por esta razón acuden a las zonas libres más próximas a su vivienda. Sin embargo los cascos antiguos, con una ocupación intensiva del espacio están generalmente muy mal dotados en espacios verdes. La creación de pequeños espacios abiertos -que no tienen que ser grandes- es una de las más importantes y urgentes conclusiones a desarrollar dentro del Casco Viejo de Logroño. 
PARQUE DE G. GALLARZA. RESUMEN TOTAL

\begin{tabular}{|c|c|c|c|c|}
\hline \multirow[t]{2}{*}{ Años de Edad } & \multicolumn{2}{|c|}{ Varones } & \multicolumn{2}{|c|}{ Mujeres } \\
\hline & N. ${ }^{\circ}$ & $\%$ s.total & N. ${ }^{\circ}$ & $\%$ s. total \\
\hline 10 & 554 & 21,64 & 512 & 22,77 \\
\hline $10-15$ & 432 & 16,90 & 396 & 17,61 \\
\hline $15-20$ & 297 & 11,61 & 145 & 6,43 \\
\hline $20-30$ & 168 & 6,57 & 289 & 12,85 \\
\hline $30-40$ & 142 & 5,55 & 234 & 10,40 \\
\hline $40-50$ & 89 & 3,48 & 131 & 5,82 \\
\hline $50-60$ & 93 & 3,63 & 96 & 4,27 \\
\hline $60-70$ & 486 & 19,01 & 289 & 13,25 \\
\hline 70 & 295 & 11,54 & 147 & 6,53 \\
\hline AI & 2556 & & 18 & \\
\hline
\end{tabular}

PARQUE DEL ESPOLON. RESUMEN TOTAL

Años de edad

10

$10-15$

$15-20$

20-30

$30-40$

$40-50$

$50-60$

$60-70$

70

TOTAL
N. ${ }^{\text {Varones }} \%$

729

501

343

214

146

124

167

919

649

3792
Mujeres

N. ${ }^{\circ} \quad \%$

$19,22 \quad 687 \quad 18,86$

$13,21 \quad 465 \quad 12,76$

$9,04 \quad 321 \quad 8,81$

$5,64 \quad 498 \quad 13,67$

$3,85 \quad 292 \quad 8,01$

$3,27 \quad 184 \quad 5,05$

$4,40 \quad 236 \quad 6,47$

$24,23 \quad 614 \quad 16,85$

$17,11 \quad 345 \quad 9,47$

3642 
PASEO DEL ESPOLON. Día 4-12-1979

Situación climática: Temp. $6^{\circ}$. Abundante niebla, mucho frío

Años de edad

10

$10-15$

$15-20$

$20-30$

$30-40$

$40-50$

$50-60$

$60-70$

70

TOTAL
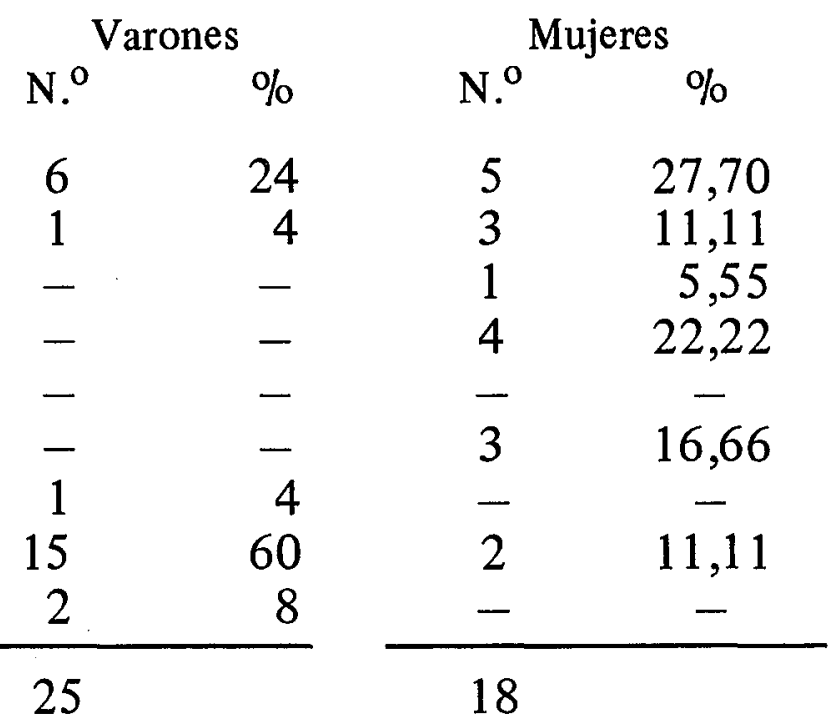

PASEO DEL ESPOLON. Día 15-6-1980

Situación climática: Temp. $22^{\circ}$. Cielo con nubles, temperatura muy agradable

Años de edad

10

10-15

15-20

20-30

$30-40$

40-50

$50-60$

60-70

70

TOTAL
N. ${ }^{\text {Varones }} \%$

$88 \quad 20,51$

$57 \quad 13,28$

$24 \quad 5,59$

46

32

20

21

98

43

10,72

7,45

4,66

4,89

22,84

10,02

429

Mujeres

N. ${ }^{\circ} \%$

76

22,02

$43 \quad 12,46$

$16 \quad 4,63$

$43 \quad 12,46$

$25 \quad 7,24$

$15 \quad 4,34$

$19 \quad 5,50$

$72 \quad 20,06$

$36 \quad 10,43$

345 
PARQUE DE GALLARZA. Día 21-XI-1979

Situación climática: Temp. $7^{\circ}$. Nublado. Mucho frío

Años de edad

\begin{tabular}{|c|c|c|c|}
\hline \multicolumn{2}{|c|}{ Varones } & \multicolumn{2}{|c|}{ Mujeres } \\
\hline $\mathrm{N}^{\circ}{ }^{\circ}$ & $\%$ & N. ${ }^{\circ}$ & $\%$ \\
\hline 18 & 24,65 & 20 & 44,44 \\
\hline 10 & 13,69 & 6 & 13,33 \\
\hline 0 & 0 & 0 & 0 \\
\hline 0 & 0 & 6 & 13,33 \\
\hline 0 & 0 & 2 & 4,44 \\
\hline 1 & 1,36 & 2 & 4,44 \\
\hline 5 & 6,84 & 2 & 2,22 \\
\hline 20 & 27,39 & 6 & 13,33 \\
\hline 19 & 26,02 & 2 & 4,44 \\
\hline 73 & & & \\
\hline
\end{tabular}

10

$10-15$

$15-20$

20-30

$30-40$

$40-50$

$50-60$

$60-70$

70

TOTAL

PARQUE DE G. GALLARZA. Día 15-6-1980

Situación climática: Temp. $20^{\circ}$. Cielo despejado. Buen día

Años de edad

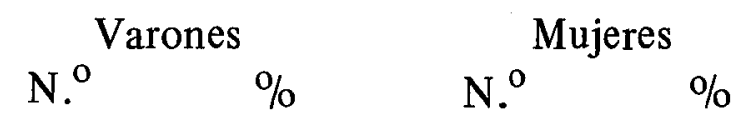

10

10-15

15-20

20-30

$30-40$

40-50

$50-60$

60-70

70

TOTAL

\begin{tabular}{|c|c|c|c|}
\hline \multicolumn{2}{|c|}{ Varones } & \multicolumn{2}{|c|}{ Mujeres } \\
\hline $\mathrm{N}^{\circ}{ }^{\circ}$ & $\%$ & N. ${ }^{\circ}$ & $\%$ \\
\hline 37 & 27,81 & 44 & 43,56 \\
\hline 20 & 15,03 & 19 & 18,81 \\
\hline 1 & & 1 & \\
\hline 6 & 4,51 & 8 & 7,92 \\
\hline 1 & & & \\
\hline 5 & 3,75 & 3 & 2,97 \\
\hline- & & 2 & 1,98 \\
\hline 55 & 41,35 & 22 & 21,78 \\
\hline 8 & 6,01 & 2 & 1,98 \\
\hline 33 & & 10 & \\
\hline
\end{tabular}




\section{LOS ESPACIOS VERDES DE LOGROÑO}

PARQUE DE G. GALLARZA. Día 28-12-1979

Situación climática: Temp. $6^{\circ}$. Cielo despejado. Día frío

Años de edad

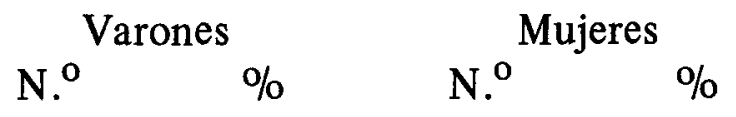

10

$10-15$

$15-20$

20-30

$30-40$

$40-50$

$50-60$

$60-70$

$36 \quad 24,32$

$35 \quad 23,64$

$6 \quad 4,05$

$2 \quad 1,33$

$1 \quad 1,35$

$1 \quad 1,35$

$26 \quad 35,60$

70

TOTAL

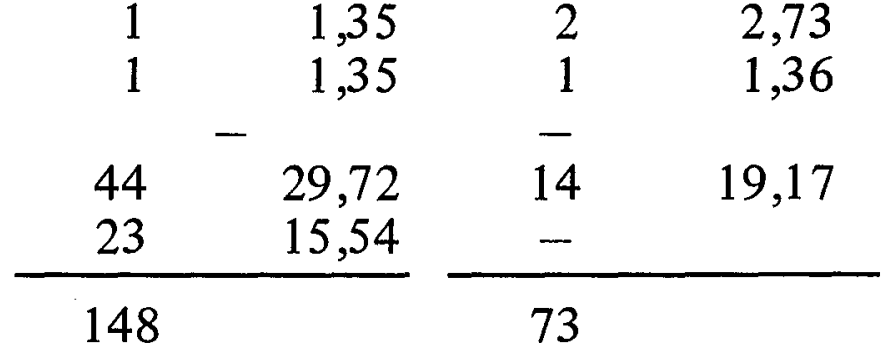

PASEO DEL ESPOLON. Día 3-1-1980

Situación climática: Temp. $8^{\circ}$. Cielo despejado, aire frío

Años de edad

N..$^{\text {Varones }} \% \quad$ N..$^{\text {Mujeres }} \%$

10

$10-15$

$15-20$

62

20,66

$64 \quad 38,55$

20-30

23

12

7,66

12

7,22

13

4

4,21

$30-40$

4,33

7

15,66

40-50

$2 \quad 0,66$

26

10,24

$50-60$

2

60-70

3

0,66

9,03

15

1

104

34,66

7

4,21

70

TOTAL

\begin{tabular}{cc}
$79 \quad 26,33$ \\
\hline 300
\end{tabular}

13

7,83

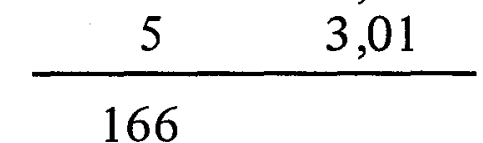


MONSERRAT MUGA FERNANDEZ

\section{BIBLIOGRAFIA}

ALONSO VELASCO, J.M., 1971: Ciudad y espacios verdes. Servicio de Publicaciones del Ministerio de la Vivienda, Madrid.

ALONSO VELASCO, J.M., 1969. Significado de los espacios verdes en la moderna cultura urbana. Rev. Ciencia Urbana, n. ${ }^{\circ} 4$, Madrid.

CAPEL SAENZ, H., 1975, Capitalismo y morfologia urbana. Ed. Los Libros de la Frontera, Barcelona.

CHAVES, R., 1973: Espacios libres y zonas verdes. Encuesta sobre la situación actual en España. Rev. Ciudad y Territorio, n. ${ }^{\circ}$ 1, Madrid.

ESTEBAN ALONSO, A., 1979: Los stándares urbanísticos. Rev. Ciudad y Territorio, $\mathrm{n}^{\circ}$ 2, Madrid.

PLAN COMARCAL DE ORDENACION URBANA DE LOGROÑO, 1975. Excmo. Ayuntamiento de Logroño.

POZO GRIJALBA, M. del, 1979: Funcionalidad y estructura interna del municipio de Logroño a través del estudio de la población. Tesis de Licenciatura inédita. Facultad de Filosofía y Letras, Zaragoza. Recientemente ha aparecido un resumen titulado "La estructura interna del municipio de Logroño: análisis por distritos administrativos: Geographicalia, 5: 71-111, Zaragoza.

SANCHEZ CASAS, C., 1971: El tiempo de ocio en la sociedad actual. Rev. Ciudad y Territorio, $\mathrm{n}^{0}$ 1, Madrid.

SANCHEZ-GABRIEL Y FERNANDEZ-GIRO, M., 1979: Climatologia y Bioclimatología aplicadas a la Rioja. Instituto de Estudios Riojanos, Logroño.

VALENZUELA RUBIO, M., 1976: Los espacios recreacionales. Rev. Ciudad y Territorio, n. $^{\circ}$, Madrid.

VALENZUELA RUBIO, M., 1979: Los espacios verdes. Rutas de BUTANO, n. ${ }^{\circ}$ 28, Universidad Autónoma de Madrid. 